\title{
Large thermoelectric power factors and impact of texturing on the thermal conductivity in polycrystalline SnSe
}

\author{
S. R. Popuri ${ }^{1}$, M. Pollet ${ }^{2,3}$, R. Decourt ${ }^{2,3}$, F. D. Morrison ${ }^{4}$, N. S. Bennett ${ }^{5}$, and J. W. G. Bos ${ }^{1}$ \\ 1. Institute of Chemical Sciences and Centre for Advanced Energy Storage and Recovery, School of \\ Engineering and Physical Sciences, Heriot-Watt University, Edinburgh, EH14 4AS, UK. \\ 2. CNRS, ICMCB, UPR 9048, Pessac F-33600, France. \\ 3. University of Bordeaux, UPR 9048, Pessac F-33600, France. \\ 4. EaStCHEM School of Chemistry, University of St Andrews, St Andrews, KY16 9ST, UK. \\ 5. Nano-Materials Lab., Institute of Mechanical, Process \& Energy Engineering, School of \\ Engineering \& Physical Sciences, Heriot-Watt University, Edinburgh EH14 4AS, UK.
}

j.w.g.bos@hw.ac.uk

Single crystals of SnSe have been reported to have very high thermoelectric efficiencies with a maximum figure merit $\mathrm{zT}=2.5$. This outstanding performance is due to ultralow thermal conductivities. We report on the synthesis of highly textured polycrystalline SnSe ingots with large single-crystal magnitude power factors, $\mathrm{S}^{2} / \rho=0.2-0.4 \mathrm{~mW} \mathrm{~m}^{-1} \mathrm{~K}^{-2}$ between $300-600 \mathrm{~K}$, increasing to $0.9 \mathrm{~mW} \mathrm{~m}^{-1} \mathrm{~K}^{-2}$ at $800 \mathrm{~K}$, and bulk thermal conductivity values $\kappa_{300 \mathrm{~K}}=1.5 \mathrm{~W} \mathrm{~m}^{-1} \mathrm{~K}^{-1}$. However, small SnSe ingots, which were measured in their entirety, were found to have a substantially reduced $\kappa_{300 \mathrm{~K}}=0.6 \mathrm{~W} \mathrm{~m}^{-1} \mathrm{~K}^{-1}$. Microscopy and diffraction revealed two distinct types of texturing within the hot-pressed ingots. In the interior, large coherent domains of SnSe platelets with a $\sim 45^{\circ}$ orientation with respect to the pressing direction are found, while the platelets are preferentially oriented at $90^{\circ}$ to the pressing direction at the top and bottom of the ingots. Fitting the $\kappa(\mathrm{T})$ data suggests an increase in defect scattering for the smaller ingots, which is in keeping with the presence of regions of structural disorder due to the change in texturing. Combining the measured $\mathrm{S}^{2} / \rho$ with the bulk ingot $\kappa$ values yields $\mathrm{zT}=1.1$ at $873 \mathrm{~K}$. 


\section{Introduction}

By some estimates two-thirds of all generated energy is lost, and the useful exploitation of waste heat is therefore of tremendous interest. ${ }^{1}$ One possible route is to produce electricity using thermoelectric generators (TEGs). These contain semiconductors and exploit a temperature difference to generate an electrical potential through the Seebeck effect. This technology has been successfully used in space exploration, where TEGs have functioned reliably for $>25$ years in the NASA Voyager deep space probes. ${ }^{2}$ However, commercial uptake has been limited by low efficiencies $(<5 \%)$ and by the high cost per kWh. ${ }^{1,3}$ The traditional thermoelectric materials are $\mathrm{Bi}_{2} \mathrm{Te}_{3}$ for near room temperature applications, and $\mathrm{PbTe}$ and $\mathrm{Si}, \mathrm{Ge}$ alloys for mid-and hightemperature applications. ${ }^{4}$ These materials have moderate efficiencies, characterised by a thermoelectric figure of merit, $\mathrm{zT}=\left(\mathrm{S}^{2} / \rho \kappa\right) \mathrm{T} \leq 1$. Here, $\mathrm{S}$ is the Seebeck coefficient $\rho$ is the electrical resistivity and $\kappa$ is the sum of the lattice $\left(\kappa_{\text {lat }}\right)$ and electronic $\left(\kappa_{\mathrm{el}}\right)$ thermal conductivities, and $\mathrm{T}$ is the absolute temperature. Over the past decades a gradual improvement has been made to the materials figure of merit. This was guided by several approaches, including the phonon-glass electron crystal concept, 5 and by nanostructuring, ${ }^{6-8}$ and has led to improvements in performance from peak $\mathrm{zT}=1$ to $\mathrm{zT}=1.5-2$ in bulk materials. ${ }^{9-12}$ In a recent significant discovery Zhao et al. demonstrated another step-change in peak zT with their measurement of zT $=2.5$ in single crystals of SnSe, convincingly breaking the $\mathrm{zT}=2$ barrier. ${ }^{13}$ This outstanding performance is due to a reported ultralow lattice thermal conductivity, $\kappa_{\text {lat }}=0.2-0.3 \mathrm{~W} \mathrm{~m}^{-1} \mathrm{~K}^{-1}$ at $800-900 \mathrm{~K}$. The thermoelectric power factor $\left(\mathrm{S}^{2} / \rho\right)$ reaches relatively modest peak values near $1 \mathrm{~mW} \mathrm{~m}^{-1} \mathrm{~K}^{-2}$ above $800 \mathrm{~K} \cdot{ }^{13}$ The crystal structure consists of two atom thick rocksalt slabs (the crystallographic bc plane), and these are displaced by c/2, leading to nearest neighbour Sn-Sn and Se-Se linkages along the a-direction. ${ }^{14,15}$ This structural anisotropy is reflected in the transport properties, where the $b$ and c directions are the most electrically and thermally conducting, while the a direction has an order of magnitude higher $\rho$, and smaller $\kappa \cdot{ }^{13,16-19}$ The low $\kappa$ results from the weak links between the rocksalt slabs and the strongly anharmonic Sn-Se bonding. ${ }^{13,20}$ At $800 \mathrm{~K}$, a phase transition 
from Pnma to an orthorhombic Cmcm structure occurs. This to leads to more symmetric rocksalt slabs, and results in a decrease of the bandgap and in an increase in carrier concentration. ${ }^{13,17,21}$ The thermal conductivity varies smoothly across the phase transition, and therefore does not appear to be strongly affected. There are now a substantial number of studies on polycrystalline SnSe samples, and none of these reproduce the $\mathrm{zT}>2$ values, with all measured $\mathrm{zT} \leq 1 .^{21-28}$ The discrepancy is linked to both the inability to reproduce the ultralow thermal conductivities, and to the reduced power factors compared to single crystals. The former is surprising as the presence of grain boundaries in polycrystalline samples usually reduces $\kappa$ compared to the single crystal values. As prepared polycrystalline $\mathrm{SnSe}$ is p-type but n-type materials have been prepared by I substitution, achieving $\mathrm{zT} \approx 0.9$ at $800 \mathrm{~K} .^{25} \mathrm{Ag}$ doping in p-type SnSe leads to a $\mathrm{zT} \approx 0.7$ at $750 \mathrm{~K}$, which is one of the highest zT values observed for polycrystalline samples due to an optimised ptype carrier concentration. ${ }^{21}$ Because of the anisotropic crystal structure, texturing of polycrystalline samples can be an important route to improve the thermoelectric properties. Many studies observe SnSe platelets, and careful orientation of these might for example be used to enhance the electrical conductivity beyond the polycrystalline average, and achieve improved zT values. We observe single-crystal magnitude thermoelectric power factors, and demonstrate that the thermal conductivity is affected by structural disorder linked to changes in texturing that occur near the boundaries of the ingots. A high-temperature structure study demonstrates the onset of significant rearrangements above $550 \mathrm{~K}$, which coincides with the maximum in the $\rho(\mathrm{T})$ data, and the increase in carrier concentration.

\section{Methods}

Polycrystalline SnSe was synthesised using standard solid state chemistry reactions. Stoichiometric amounts of 100-mesh Sn (99.9\%, Alfa Aesar) and Se powder (99.999\%, Alfa Aesar) were ground together and cold pressed into a pellet. The pellet was sealed in an evacuated quartz tube to $10^{-5}$ bar and heated at $900{ }^{\circ} \mathrm{C}$ for $18 \mathrm{~h}\left(10{ }^{\circ} \mathrm{C} \mathrm{min}^{-1}\right.$ heating ramp), followed by cooling at $3{ }^{\circ} \mathrm{C} \min ^{-1}$ to 
room temperature. This heating step was repeated once with an intermediate homogenisation. Synthesis temperatures higher than $900{ }^{\circ} \mathrm{C}$ resulted in the formation of a black residue on the walls of the tubes. The sintered pellets were ground to powders and hot-pressed into $13 \mathrm{~mm}$ diameter cylindrical ingots under Ar atmosphere for $20 \mathrm{~min}$ at $500{ }^{\circ} \mathrm{C}$ and $60 \mathrm{MPa}$. Photographs of the ingots are shown in Fig. S1 of the electronic supplementary information. The hot-press is an homebuilt instrument exploiting induction heating and is based on the design of Ref. 29. The phase purity of the samples was confirmed on powder specimens and pieces cut from the hot-pressed ingots using a Bruker D8 Advance diffractometer with monochromated $\mathrm{Cu} \mathrm{K}_{\alpha 1}$ radiation. The variable temperature structural study was done using a PANalytical Empyrean diffractometer $\left(\mathrm{Mo} \mathrm{K}_{\alpha 1, \alpha 2}\right)$ fitted with an Anton-Paar furnace. Rietveld analysis was undertaken using the GSAS/EXPGUI suite of programmes. ${ }^{30,31}$ The microstructure and elemental distributions were analysed using a Quanta 650 FEG SEM fitted with an Oxford Instruments X-max $150^{\mathrm{N}}$ detector. The temperature dependence of the electrical resistivity and the Seebeck coefficient were measured using a Linseis LSR-3 instrument. A diamond saw was used to obtain rectangular bars from the hot-pressed ingots. The thermal diffusivity (D) and heat capacity $\left(\mathrm{C}_{\mathrm{p}}\right)$ were measured using a Netzch LFA 457 and Perkin Elmer DSC 8500, respectively. The thermal conductivity was calculated using: $\kappa=$ $\alpha(\mathrm{T}) \mathrm{C}_{\mathrm{p}}(\mathrm{T}) \rho$, where $\rho$ is the crystallographic density, $6.18 \mathrm{~g} \mathrm{~cm}^{-3}$. The relative density of all the samples is ca. $98 \%$. The lattice thermal conductivity was estimated using the Wiedemann-Franz law: $\kappa_{\text {lat }}=\kappa-\mathrm{L} \sigma \mathrm{T}$, where $\mathrm{L}$ is the Lorenz number $\left(2.44 \times 10^{-8} \mathrm{~V}^{2} \mathrm{~K}^{-2}\right)$. Room temperature Hall effect measurements were made in Van der Pauw geometry, using a Biorad HL5900 tool. Room temperature thermal conductivity measurements were made using a Thermal Transport Option fitted in a Quantum Design Physical Property Measurement System.

\section{Results}


We prepared two 2.5 gram (ingot \#1,2) and one 11 gram SnSe ingot (\#3), and measured the thermoelectric properties parallel $(/ /)$ and perpendicular $(\perp)$ to the hot-press direction, as illustrated in the inset to Fig. 2. The ingot heights were $3 \mathrm{~mm}$ for \#1, 2 and $13 \mathrm{~mm}$ for ingot \#3.
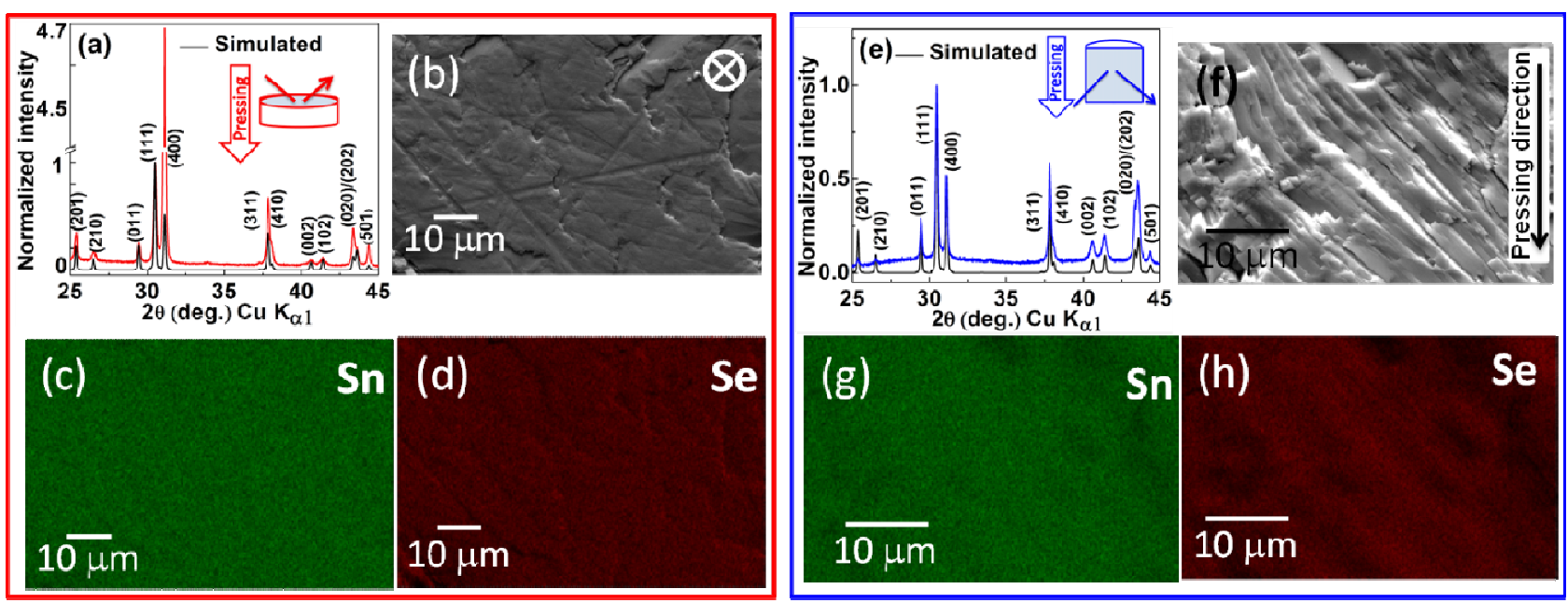

Fig. 1. Representative X-ray powder diffraction patterns, SEM micrographs and EDX elemental maps for Sn and Se acquired on surfaces perpendicular (a-d) and parallel (e-f) to the hot-press direction. Simulated XRD patterns illustrating the powder average are included for comparison. Data collected on ingot \#1.

Structure. The structural and morphological characterisation of a representative ingot (\#1) is summarised in Fig. 1. X-ray diffraction data were collected on a face perpendicular (Fig. 1a) and parallel (Fig. 1e) to the hot-press direction. No evidence for any other phases than SnSe was observed, and clear evidence for texturing was evident in the data. In particular, the (400) reflection is strongly enhanced for the data collected on the top face of the ingot (Fig. 1a), while the (111) reflection is over represented for the face parallel to the hot-press direction (Fig. 1e). We analysed the texturing using a March-Dollase preferred orientation function. ${ }^{30}$ For the data collected on the top face this suggested the presence of thin-platelets with a high degree of texturing (the h00 are enhanced 2.5 times compared to the powder average). This is in keeping with the SEM data in Fig. $1 \mathrm{~b}$ which reveal a flat surface, in keeping with a top down view of platelets. The texturing for the face parallel to the pressing direction (Fig. 1e) is not very well fitted using a single (111) preferred 
orientation direction, evidencing a less straightforward arrangement of the SnSe platelets with respect to the pressing direction. The SEM data are very insightful here, and reveal large domains of $\sim 45^{\circ}$ oriented 0.7-1 $\mu \mathrm{m}$ thickness SnSe platelets with typical lengths $20-30 \mu \mathrm{m}$. This suggests a preferential crystallite growth in the bc plane with the a-direction perpendicular to the platelets. ${ }^{21,22,}$ ${ }^{25,26}$ These $\sim 45^{\circ}$ domains are a universal feature of all prepared ingots. From a large number of images collected on ingots 1 and 3 we have observed angles between $43-52^{\circ}$. The similarity between ingots is confirmed by the texturing observable from diffraction, as illustrated by the identical patterns for ingots 1 and 3 in Fig. S2 of the electronic supplementary information (ESI). The change in texturing from SnSe platelets at $90^{\circ}$ to the pressing direction at the surface to $45^{\circ}$ in the interior is striking. Similar texturing is evident in the literature but the areas of uniform stacking appear to be larger for our ingots..$^{21,22,25,26}$ The change in texturing from $\sim 90^{\circ}$ to $\sim 45^{\circ}$ is also apparent from SEM data collected along an $8 \mathrm{~mm}$ bar cut from ingot \#3 (Figure S3 in the ESI). The parts of the bar that were part of the interior of the ingot show the large coherent $45^{\circ}$ domains, while the image collected near the bottom of the ingot shows greatly enhanced disorder, consistent with a change in dominant texturing to a $90^{\circ}$ orientation. The elemental mapping reveals a homogenous distribution of $\mathrm{Sn}$ and Se without any evidence for the presence of elemental $\mathrm{Sn}$ as observed elsewhere. ${ }^{22}$ The EDX compositions are identical for the three ingots and are $\mathrm{Sn}: \mathrm{Se}=$ 0.50(1):0.49(1), in keeping with a stoichiometric composition. Rietveld analysis indicated fully occupied $\mathrm{Sn}$ and Se sites. The structural analysis therefore indicates that $\mathrm{SnSe}$ is phase pure and stoichiometric, and that there are large domains of stacked SnSe platelets with a $\sim 45^{\circ}$ orientation with respect to the hot-press direction in the interior of the ingots. 

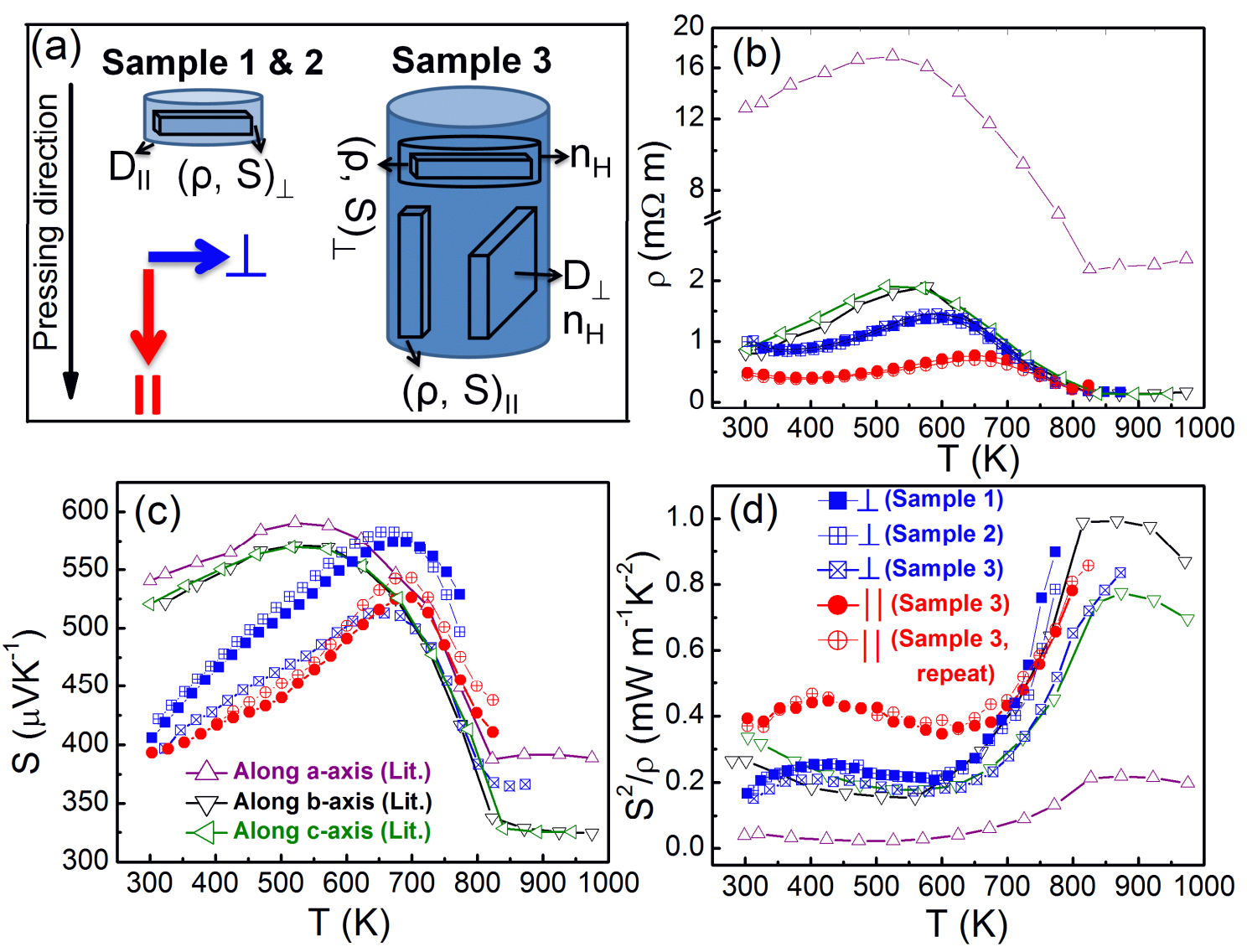

Fig. 2. (a) Overview of the prepared SnSe ingots and the bars and disks used for the individual measurements. Temperature dependence of (b) the electrical resistivity ( $\rho)$; (c) the Seebeck coefficient (S); and (d) the thermoelectric power factor $\left(S^{2} / \rho\right)$. Single crystal data from Ref. ${ }^{13}$ are included for comparison.

Electrical Transport. The temperature dependences of $\rho, S$ and $S^{2} / \rho$ are shown in Fig. 2. The single crystal data from Ref. 13 are included for comparison. The electrical transport measurement were repeated at least twice on each bar, and we tested several pieces of each ingot. These experiments yielded similar results, demonstrating that our electrical transport data are reproducible. The overall Curie symmetry of the cylindrical ingot is $\infty / \mathrm{mm}$, and we therefore expect anisotropy in the measured physical properties for measurements // and $\perp$ to the hot-press direction. Any differences observed in the // and $\perp$ directions reflect a different weighting of the single crystal directions, and also include grain boundary contributions. The data for the $\perp$ direction are the average of two repeat 
measurements, while both datasets are shown for // direction, illustrating the excellent reproducibility. There are only minor differences between the small and large ingots, in particular the maximum $\mathrm{S}(\mathrm{T})$ values are somewhat lower for the large ingot. However, on the whole the measured transport properties are similar, suggesting little difference in chemical composition and texturing between ingots, and thus confirming the reproducibility of our synthesis and consolidation procedure. The most striking result are the very low $\rho(T)$ values compared to the single crystal data (Fig. 2b). ${ }^{13}$ Assuming that an ideal $45^{\circ}$ orientation of SnSe platelets holds throughout we expect $\rho_{/ /}$ and $\rho_{\perp}$ to have similar magnitudes, which includes out-of-plane $\left(\propto \rho_{\mathrm{b}, \mathrm{c}}\right)$ and in-plane $\left(\propto \rho_{\mathrm{a}}\right)$ components and a contribution due to boundary resistances. The polycrystalline average of the single crystal data $\rho_{300 \mathrm{~K}}=500 \mathrm{~m} \Omega \mathrm{cm}$, while the average of the $/ /$ and $\perp$ directions is $75 \mathrm{~m} \Omega \mathrm{cm}$. This 6-7 fold reduction is linked to an increased carrier concentration as is evident from the $\mathrm{S}(\mathrm{T})$ data and Hall measurements (see below). Compared to other polycrystalline samples, these low $\rho(\mathrm{T})$ values are unprecedented. ${ }^{20,21,24-26}$ Typical literature values for the // and $\perp$ directions fall between $700-1500 \mathrm{~m} \Omega \mathrm{cm}$ at $300 \mathrm{~K}$, whereas the values reported here are an order of magnitude lower (Fig. 2b). The room temperature Seebeck values for our ingots are near $400 \mu \mathrm{V} \mathrm{K}^{-1}$, which is $\sim 125 \mu \mathrm{V} \mathrm{K}^{-1}$ smaller than the single crystal data (Fig. 2c), ${ }^{13}$ suggesting a higher p-type carrier concentration. Hall measurements yielded identical concentrations of $4.0(2) \times 10^{17}$ holes $^{-3}$ for disks cut // and $\perp$ to the hot-press direction. This concentration is larger than reported for other polycrystalline SnSe samples, e.g. $\sim 2.5 \times 10^{17}$ holes $\mathrm{cm}^{-3}$ in Refs. 21,25 with $\mathrm{S}_{300 \mathrm{~K}}=500-600 \mu \mathrm{V}$ $\mathrm{K}^{-1}$. We attribute the increased p-type carrier concentration to a stoichiometric composition, and the absence of excess Sn, which has been linked with n-type compensating defects. ${ }^{21}$ The higher carrier concentration leads to large $\mathrm{S}^{2} / \rho$ values in the Pnma phase: $0.2-0.4 \mathrm{~mW} \mathrm{~m}^{-1} \mathrm{~K}^{-2}$ from $300-600 \mathrm{~K}$, increasing to $0.9 \mathrm{~mW} \mathrm{~m}^{-1} \mathrm{~K}^{-2}$ at $800 \mathrm{~K}$ (Fig. 2d). At high temperatures the measured $\mathrm{S}(\mathrm{T})$ and $\rho(\mathrm{T})$ are comparable to the single crystal data and to other studies on polycrystalline $\mathrm{SnSe}$. This consistency between all studies is in keeping with the order of magnitude increase in carrier 
concentration with temperature, ${ }^{13,21}$ which rapidly quenches any n-type compensation due to variations in sample processing. In the $\mathrm{Cmcm}$ phase, there is negligible anisotropy for measurements // and $\perp$ to the pressing direction, which signals the decreasing influence of boundary scattering at high-temperatures. The absence of anisotropy is in keeping with the dominant $45^{\circ}$ texturing.

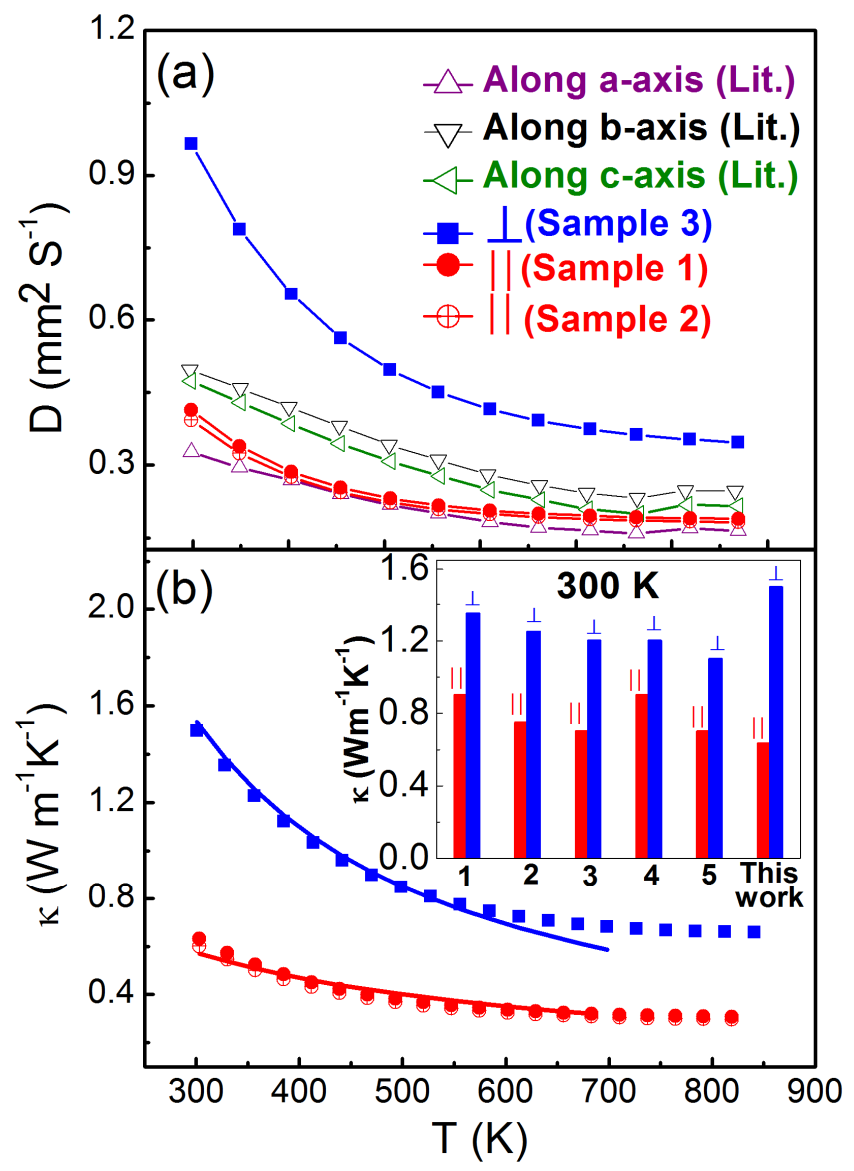

Fig. 3. Temperature dependence of (a) the thermal diffusivity (D) and (b) the thermal conductivity

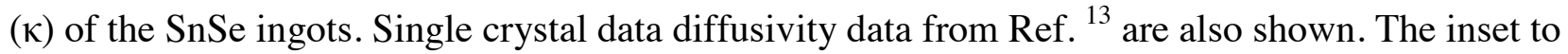
panel (b) gives a comparison of $\kappa_{300 \mathrm{~K}}$ values from the literature (sample $1^{21} ; 2^{22} ; 3^{27} ; 4^{26}, 5^{25}$ ). The solid lines to the $\kappa(\mathrm{T})$ data are fits to a Callaway model, and are discussed in the text of the manuscript.

Thermal transport: The temperature dependence of the thermal diffusivity (D) and thermal conductivity ( $\kappa)$ are shown in Fig. 3. D(T) data were collected in the // directions on ingots 1,2 and 
in the $\perp$ direction for ingot 3 (see Fig. 2a). The heat capacity data are shown in Fig. S4 of the ESI. The electronic contribution to $\kappa$ remains small (Fig. S5 of the ESI). The $\mathrm{D}(\mathrm{T})$ and $\kappa(\mathrm{T})$ values for the smaller ingots are close to the polycrystalline average of the single crystal data, ${ }^{13}$ while the bigger ingot yielded much larger $\kappa$ values, e.g. $\kappa_{300 \mathrm{~K}}=1.5 \mathrm{~W} \mathrm{~m}^{-1} \mathrm{~K}^{-1}$ at $300 \mathrm{~K}$ compared to $0.6 \mathrm{~W}$ $\mathrm{m}^{-1} \mathrm{~K}^{-1}$ for the smaller ingots. This large anisotropy is unexpected as the $\rho(\mathrm{T})$ data suggested that both measurements directions should yield similar results, and this may therefore suggest strongly varying boundary resistances. The observed values are consistent with the literature on polycrystalline samples, as shown in the inset to Fig. $3 \mathrm{~b}$. In all cases, $\kappa_{/ /}<1 \mathrm{~W} \mathrm{~m}^{-1} \mathrm{~K}^{-1}$, while $\kappa_{\perp}>1$ $\mathrm{W} \mathrm{m} \mathrm{m}^{-1} \mathrm{~K}^{-1}$ at $300 \mathrm{~K}$. In fact our values are at the upper and lower limit compared to the other published data. In particular the low $\kappa_{/ /}$are of interest and suggest that large zT values might be possible. In order to understand the cause of the large discrepancy we fitted the $\kappa(\mathrm{T})$ data to a modified Callaway model with contributions due to boundary, point defect and Umklapp phonon scattering: $:^{32}$

$$
\begin{gathered}
\kappa=\frac{k_{B}}{2 \pi^{2} v_{s}}\left(\frac{k_{B} T}{\hbar}\right)^{3} \int_{0}^{\theta D / T} \frac{x^{4} e^{x}}{\tau_{p h}^{-1}\left(e^{x}-1\right)^{2}} d x \\
\text { with } \tau_{p h}^{-1}=\tau_{B}^{-1}+\tau_{P D}^{-1}+\tau_{U}^{-1}=\frac{V_{s}}{L}+A \omega^{4}+B \omega^{2} T e^{-\theta_{D} / 3 T}
\end{gathered}
$$

Here, $x=\hbar \omega / k_{B} T$ is the reduced phonon frequency, $\hbar$ is the reduced Planck constant, $k_{B}$ is the Boltzmann constant, $\mathrm{v}_{\mathrm{S}}=2000 \mathrm{~m} \mathrm{~s}^{-1}$ is the speed of sound, $\theta_{\mathrm{D}}=210 \mathrm{~K}$ is the Debye temperature, ${ }^{33}$ and $\mathrm{T}$ is the absolute temperature. The fitting parameters are $\mathrm{L}$ for the average grain size, $\mathrm{A}$ for the magnitude of point defect scattering, and B for the magnitude of the Umklapp scattering. Boundary scattering is usually only significant at low temperatures and $\mathrm{L}=10 \mu \mathrm{m}$ was used here. A comparison of the best fit parameters is shown in Table 1, and the fits are shown in Fig. 3b. The larger $\kappa_{\perp}$ can be fitted between 300 and $700 \mathrm{~K}$ using an Umklapp phonon scattering contribution. Least squares fitting of the $\kappa_{/ /}$indicates a similar Umklapp scattering strength but now augmented 
by an additional point defect scattering contribution. In the absence of intentional alloying the cause of this additional contribution is unclear but will be discussed below.

Table 1. Final fitted parameters used in Callaway's model. A and B are point defect and Umklapp scattering parameters, $\mathrm{L}$ is the average grain size.

\begin{tabular}{lll}
\hline & $\kappa_{\perp}$ & $\kappa_{\|}$ \\
\hline $\mathrm{A}\left(\mathrm{s}^{3}\right)$ & 0 & $9.725 \times 10^{-41}$ \\
$\mathrm{~B}\left(\mathrm{~s} \mathrm{~K}^{-1}\right)$ & $2.50 \times 10^{-17}$ & $2.56 \times 10^{-17}$ \\
$\mathrm{~L}(\mu \mathrm{m})$ & 10 & 10 \\
\hline
\end{tabular}

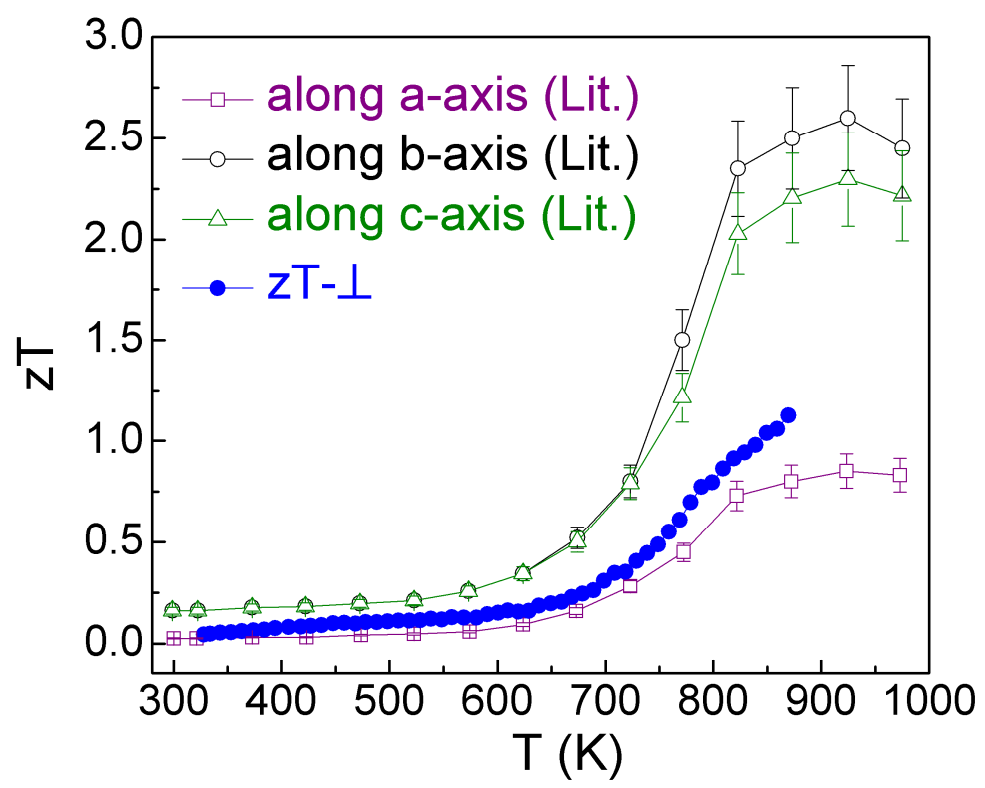

Fig. 4. Temperature dependence of the thermoelectric figure of merit (zT) using data collected on ingot \#3 in the $\perp$-direction. Literature data for SnSe single crystals are also shown. ${ }^{13}$

Figure of merit. The temperature dependence of zT for the $\perp$-direction was obtained from data collected on ingot \#3 and is shown in Fig. 4. This reveals zT $=0.8$ at $800 \mathrm{~K}$, increasing to $\mathrm{zT}=1.1$ at $873 \mathrm{~K}$. We were not able to measure $\left(\mathrm{S}^{2} / \rho\right)_{/ /}$directly on ingot \#1,2 due to their small height and are therefore not able to calculate $\mathrm{zT}_{/ /}$for the small ingots with the low $\kappa$ values. However, the similar $\left(S^{2} / \rho\right)_{\perp}$ values for the small and large ingots (Fig. 2) suggest that the $\left(S^{2} / \rho\right)_{/ /}$for the small ingots could also be large, and that large zT values may be possible for that direction. 

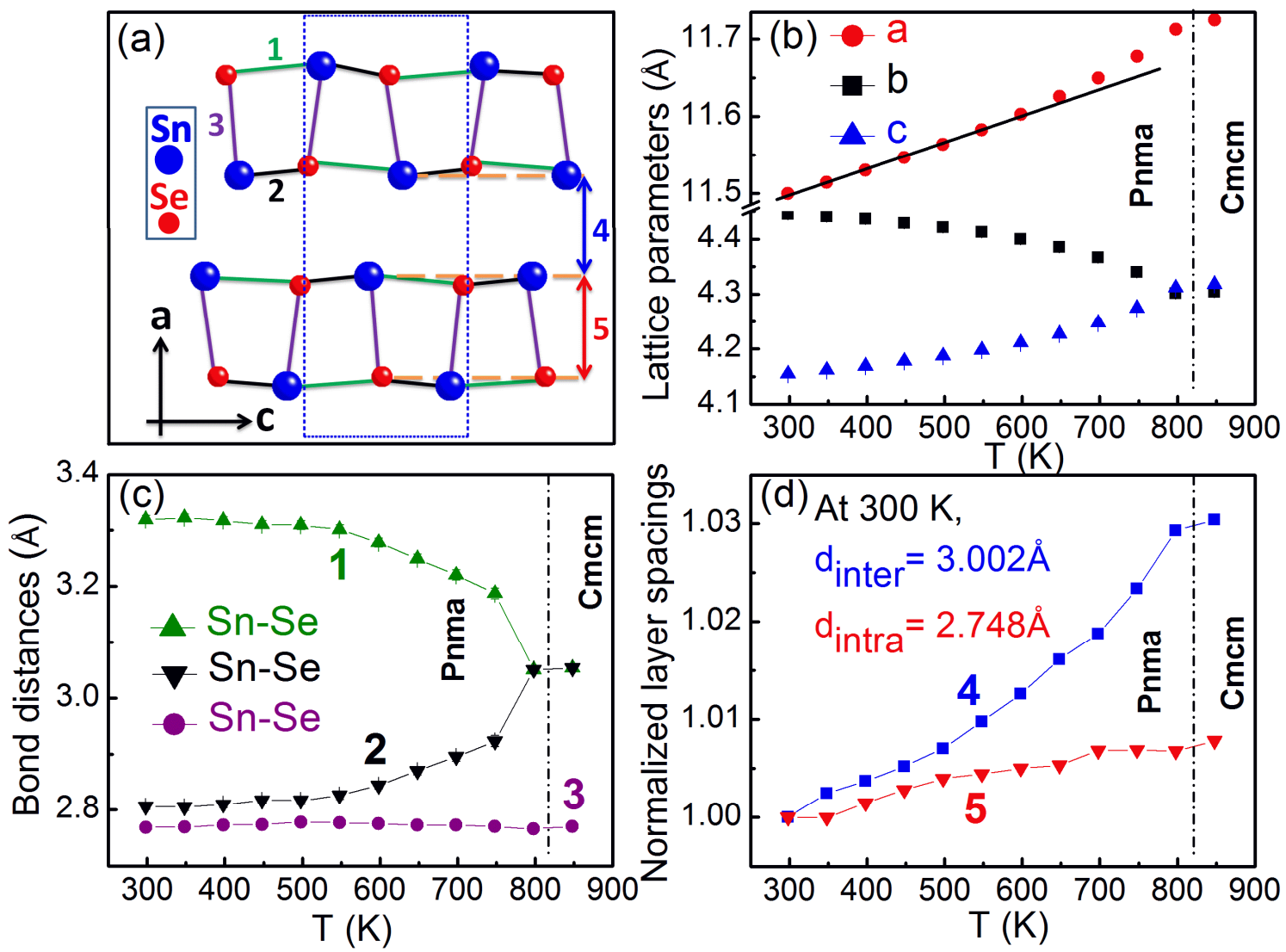

Fig. 5. (a) View of the crystal structure of SnSe (Pnma setting), indicating unique Sn-Se bond distances (1-3) and the inter (4) and intra (5) layer spacing. The temperature dependence of (b) the lattice parameters, (c) selected Se-Sn bond distances and (d) the inter- and intralayer spacing. The black line in panel (b) is a guide to the eye.

Variable temperature structural study. SnSe undergoes a structural phase transition from Pnma $(\mathrm{a}=$ $11.50 \AA, b=4.45 \AA, c=4.15 \AA)$ to $\mathrm{Cmcm}(\mathrm{a}=4.32 \AA, \mathrm{b}=11.70 \AA, \mathrm{c}=4.32 \AA)$ at $800 \mathrm{~K} \cdot{ }^{14,15} \mathrm{~A}$ projection of the crystallographic a-c plane of the Pnma structure is shown in Fig. 5a. A specimen Rietveld fit (Fig. S6) and a table with refined crystallographic data can be found in the ESI (Table S1). The temperature dependence of the lattice parameters is shown in Fig. 5b, and reveals an almost linear expansion of the a-axis up to $550 \mathrm{~K}$, beyond which a more rapid increase is observed. Similar deviations from linear temperature dependence above $550 \mathrm{~K}$ are observed for the b- and cdirections, which approach each other, and attain similar values in the $\mathrm{Cmcm}$ structure (Fig. 5b). The deviation from linear expansion is also evident in some of the Sn-Se bond distances (Fig. 5c) 
and in the distance between the SnSe rocksalt slabs (interlayer spacing in Fig. 5d). By contrast the SnSe intralayer distance increases linearly (Fig 5d). Inspection of the structural data reveals that temperature gradually forces $\mathrm{Sn}$ and $\mathrm{Se}$ in adjacent rocksalt slabs to be on top of each other (they attain the same $\mathrm{z}$-coordinate in the $\mathrm{Cmcm}$ structure), leading to an accelerated (non-linear) expansion of the inter layer distance (Fig. 5 d). The impact of temperature is quite different from pressure, where the intra and interlayer distances become equal at $\sim 11 \mathrm{GPa}^{34}$ In terms of the thermoelectric properties, both $\rho(\mathrm{T})$ and $\mathrm{S}(\mathrm{T})$ decrease rapidly above $600-650 \mathrm{~K}$ (Fig. 2b). This temperature corresponds closely with the onset of the structural phase transition as determined here. The link between the structural changes $<800 \mathrm{~K}$ and the drop in $\mathrm{S}(\mathrm{T})$ and $\rho(\mathrm{T})$ is supported by density functional calculations. These reveal significant changes to the band dispersion within the Pnma phase above $550 \mathrm{~K}$, leading to an increased carrier concentration, ${ }^{17}$ which is consistent with the observed decrease in $S(T)$ and $\rho(T)$.

\section{Discussion}

There are now a number of reports on the thermoelectric properties of polycrystalline SnSe in the literature. ${ }^{21-28}$ None of these approaches the large zT values in single crystals that sparked the renewed interest in this system. We have succeeded in imparting large $S^{2} / \rho$, in particular within the Pnma phase, and have found low $\kappa$ values for small ingots that were measured in their entirety, while disks cut from the interior of the ingots have larger $\kappa$ values. During review of this manuscript two articles appeared using $\mathrm{Na}$ to intentionally p-type dope $\mathrm{SnSe}$, leading to large power factors within the Pnma phase, and record device ZTs in single crystals. ${ }^{35,36}$ This supports our conclusion of improved $\mathrm{S}^{2} / \rho$ due to an increased hole concentration. SEM and X-ray diffraction reveal a textured arrangement of SnSe platelets that are oriented at $\sim 45^{\circ}$ with respect to the pressing direction in the interior of the ingots. The texturing near the top and bottom faces is different with a dominant $90^{\circ}$ alignment of the SnSe plates. In an ideal model with a $45^{\circ}$ orientation of the platelets, identical in-plane and out-of-plane contributions are expected for measurements $/ /$ and $\perp$ to the 
pressing direction. The only difference should be due to the number of times the electron and phonons pass between platelets. Experimentally, nearly identical $\rho(\mathrm{T})$ are observed as the Cmcm phase is approached, while the 2-fold anisotropy at lower temperatures is consistent with different boundary contributions. Based on the $\rho(\mathrm{T})$ data, similar $\kappa(\mathrm{T})$ are also expected for measurements // and $\perp$ to the hot-pressing direction. However, the experimental data suggest that this is not the case. There is considerable uncertainty in the literature regarding the intrinsic $\kappa$ of $\mathrm{SnSe}$ with computational, ${ }^{18,19}$ and earlier single crystal studies indicating in-plane values up to $2 \mathrm{~W} \mathrm{~m}^{-1} \mathrm{~K}^{-1}$. 37 , ${ }^{38}$ To confirm our values we measured the room temperature $\kappa$ on bars cut from the large ingot (\#3) using a Quantum Design Thermal Transport Option. These confirmed $\kappa_{\perp}=1.5 \mathrm{~W} \mathrm{~m}^{-1} \mathrm{~K}^{-1}$ but yielded $\kappa_{/ /}=1.8 \mathrm{~W} \mathrm{~m}^{-1} \mathrm{~K}^{-1}$, compared to $0.6 \mathrm{~W} \mathrm{~m}^{-1} \mathrm{~K}^{-1}$ observed on the two smaller ingots. This suggests that $\kappa$ within the interior of the pellets is in fact nearly identical for both measurement directions. Modelling of $\kappa(\mathrm{T})$ using a Callaway model indicated dominant phonon-phonon scattering for the disk cut from the interior of the ingot, whereas the smaller ingots have an additional contribution due to point defect scattering. The texturing and compositions of the large and smaller ingots are very similar. The only difference is that the smaller ingots were measured in their entirety, and therefore also include the boundary regions where a change of texturing is evident. It is conceivable that these regions of increased structural disorder (e.g. image 4 in Fig. S3 in the ESI) can act as additional phonon scattering centres, and cause a reduction in $\kappa$. Recent theoretical work suggests that point defect scattering of optic phonons can reduce $\kappa$ by up to $60 \%$ which would correspond to $0.6-0.8 \mathrm{~W} \mathrm{~m}^{-1} \mathrm{~K}^{-1}$ at $300 \mathrm{~K} .{ }^{18}$ The low $\kappa_{/ /}$for the smaller ingots cannot be explained by a different averaging of the single crystal directions. Unfortunately it is not possible to undertake a 4-point measurement that includes the boundary regions and confirm that the large $\mathrm{S}^{2} / \rho$ carry over to the smaller ingots. Two-point resistance measurements yielded similar values for both measurement directions suggesting that the carrier transport is not strongly affected by the boundary regions. This indicates that large figures of merit may be possible, provided that the 
microstructure of these materials can be engineered appropriately to unite the large $S^{2} / \rho$ and low $\kappa$. Because of the difficulty in accurately combining the measured parameters, demonstration of high thermoelectric efficiencies will rely on the integration of SnSe in a working thermoelectric generator device.

\section{Acknowledgements}

This work was supported by the Leverhulme Trust (RPG-2012-576). Jim Buckman, Institute of Petroleum Engineering, Heriot-Watt University, is acknowledged for his help during collection of the SEM data.

\section{Notes}

Electronic Supplementary Information (ESI) available: XRD patterns, additional SEM images, specific heat, lattice and electronic thermal conductivity, and variable temperature structural data. See DOI: $10.1039 / \mathrm{x} 0 \mathrm{xx} 00000 \mathrm{x}$

\section{Competing Financial Interests}

The authors declare no competing financial interests.

\section{References}

1. D. M. Rowe, ed., Thermoelectrics and its Energy Harvesting, CRC Press Boca Raton, 2012.

2. J. H. Yang and T. Caillat, MRS Bulletin, 2006, 31, 224-229.

3. S. LeBlanc, S. K. Yee, M. L. Scullin, C. Dames and K. E. Goodson, Renewable and Sustainable Energy Reviews, 2014, 32, 313-327.

4. G. J. Snyder and E. S. Toberer, Nature Materials, 2008, 7, 105-114.

5. $\quad$ B. C. Sales, D. Mandrus and R. K. Williams, Science, 1996, 272, 1325-1328.

6. A. J. Minnich, M. S. Dresselhaus, Z. F. Ren and G. Chen, Energy \& Environmental Science, 2009, 2, 466-479.

7. M. G. Kanatzidis, Chemistry of Materials, 2009, 22, 648-659.

8. C. J. Vineis, A. Shakouri, A. Majumdar and M. G. Kanatzidis, Advanced Materials, 2010, 22, 3970-3980.

9. K. Biswas, J. He, I. D. Blum, C. I. Wu, T. P. Hogan, D. N. Seidman, V. P. Dravid and M. G. Kanatzidis, Nature, 2012, 489, 414-418. 
10. K. Biswas, J. He, Q. Zhang, G. Wang, C. Uher, V. P. Dravid and M. G. Kanatzidis, Nature Chemistry, 2011, 3, 160-166.

11. K. F. Hsu, S. Loo, F. Guo, W. Chen, J. S. Dyck, C. Uher, T. Hogan, E. K. Polychroniadis and M. G. Kanatzidis, Science, 2004, 303, 818-821.

12. B. Poudel, Q. Hao, Y. Ma, Y. C. Lan, A. Minnich, B. Yu, X. A. Yan, D. Z. Wang, A. Muto, D. Vashaee, X. Y. Chen, J. M. Liu, M. S. Dresselhaus, G. Chen and Z. F. Ren, Science, 2008, 320, 634-638.

13. L. D. Zhao, S. H. Lo, Y. Zhang, H. Sun, G. Tan, C. Uher, C. Wolverton, V. P. Dravid and M. G. Kanatzidis, Nature, 2014, 508, 373-377.

14. H. G. Vonschnering and H. Wiedemeier, Zeitschrift Fur Kristallographie, 1981, 156, $143-$ 150.

15. T. Chattopadhyay, J. Pannetier and H. G. Vonschnering, Journal of Physics and Chemistry of Solids, 1986, 47, 879-885.

16. G. Shi and E. Kioupakis, Journal of Applied Physics, 2015, 117, 065103.

17. K. Kutorasinski, B. Wiendlocha, S. Kaprzyk and J. Tobola, Physical Review B, 2015, 91, 205201.

18. R. Q. Guo, X. J. Wang, Y. D. Kuang and B. L. Huang, Physical Review B, 2015, 92, 115202.

19. J. Carrete, N. Mingo and S. Curtarolo, Applied Physics Letters, 2014, 105, 101907.

20. C. W. Li, J. Hong, A. F. May, D. Bansal, S. Chi, T. Hong, G. Ehlers and O. Delaire, Nature Physics, 2015, doi:10.1038/nphys3492.

21. C.-L. Chen, H. Wang, Y.-Y. Chen, T. Day and G. J. Snyder, Journal of Materials Chemistry A, 2014, 2, 11171.

22. S. Sassi, C. Candolfi, J. B. Vaney, V. Ohorodniichuk, P. Masschelein, A. Dauscher and B. Lenoir, Applied Physics Letters, 2014, 104, 212105.

23. C. Bera, S. Jacob, I. Opahle, N. S. H. Gunda, R. Chmielowski, G. Dennler and G. K. H. Madsen, Physical Chemistry Chemical Physics, 2014, 16, 19894-19899.

24. F. Serrano-Sanchez, M. Gharsallah, N. M. Nemes, F. J. Mompean, J. L. Martinez and J. A. Alonso, Applied Physics Letters, 2015, 106, 083902.

25. Q. Zhang, E. K. Chere, J. Sun, F. Cao, K. Dahal, S. Chen, G. Chen and Z. Ren, Advanced Energy Materials, 2015, 5, 1500360.

26. Y. Li, X. Shi, D. Ren, J. Chen and L. Chen, Energies, 2015, 8, 6275-6285.

27. Y.-M. Han, J. Zhao, M. Zhou, X.-X. Jiang, H.-Q. Leng and L.-F. Li, J. Mater. Chem. A, $2015,3,4555-4559$.

28. Z. H. Ge, K. Y. Wei, H. Lewis, J. Martin and G. S. Nolas, Journal of Solid State Chemistry, 2015, 225, 354-358.

29. A. D. LaLonde, T. Ikeda and G. J. Snyder, Review of Scientific Instruments, 2011, 82, 025104.

30. A. C. Larson and R. B. Von Dreele, in Los Alamos National Laboratory Report LAUR, 2000, pp. 86-748.

31. B. H. Toby, Journal of Applied Crystallography, 2001, 34, 210-213.

32. J. Callaway, Physical Review, 1959, 113, 1046-1051.

33. O. Madelung, Semiconductors: Data Handbook, Springer-Verlag, Berlin Heidelberg, 2004.

34. I. Loa, R. J. Husband, R. A. Downie, S. R. Popuri and J. W. G. Bos, Journal of Physics: Condensed Matter, 2015, 27, 072202.

35. L.-D. Zhao, G. Tan, S. Hao, J. He, Y. Pei, H. Chi, H. Wang, S. Gong, H. Xu, V. P. Dravid, C. Uher, G. J. Snyder, C. Wolverton and M. G. Kanatzidis, Science, 2016, 351, 141-144.

36. K. Peng, X. Lu, H. Zhan, S. Hui, X. Tang, G. Wang, J. Dai, C. Uher, G. Wang and X. Zhou, Energy \& Environmental Science, 2016, 10.1039/C1035EE03366G.

37. J. D. Wasscher, W. Albers and C. Haas, Solid-State Electronics, 1963, 6, 261-264.

38. D. P. Spitzer, Journal of Physics and Chemistry of Solids, 1970, 31, 19. 
Supplementary Information for:

\section{Large thermoelectric power factors in polycrystalline $\mathrm{SnSe}$}

S. R. Popuri ${ }^{1}$, M. Pollet ${ }^{2,3}$, R. Decourt ${ }^{2,3}$, F. D. Morrison ${ }^{4}$, N. S. Bennett ${ }^{5}$, and J. W. G. Bos ${ }^{1}$

1. Institute of Chemical Sciences and Centre for Advanced Energy Storage and Recovery, School of Engineering and Physical Sciences, Heriot-Watt University, Edinburgh, EH14 4AS, UK.

2. CNRS, ICMCB, UPR 9048, Pessac F-33600, France.

3. University of Bordeaux, UPR 9048, Pessac F-33600, France.

4. EaStCHEM School of Chemistry, University of St Andrews, St Andrews, KY16 9ST, UK.

5. Nano-Materials Lab., Institute of Mechanical, Process \& Energy Engineering, School of Engineering \& Physical Sciences, Heriot-Watt University, Edinburgh EH14 4AS, UK.

Table S1: Refined lattice and atomic parameters for SnSe. Data obtained from Rietveld fits against X-ray powder diffraction data.

\begin{tabular}{|c|c|c|c|c|c|c|c|c|c|}
\hline \multirow{2}{*}{$\begin{array}{c}\mathbf{T} \\
(\mathbf{K})\end{array}$} & \multicolumn{3}{|c|}{ Lattice parameters $(\AA)$} & \multicolumn{2}{|c|}{ Sn } & \multicolumn{2}{|c|}{ Se } & \multirow{2}{*}{$\begin{array}{c}\mathbf{1 0 0}^{*} \\
\mathbf{U}_{\text {iso }} \\
\left(\AA^{2}\right)\end{array}$} & \multirow{2}{*}{$\chi^{2}$} \\
\hline & $\mathbf{a}$ & b & c & $\mathbf{x}$ & $\mathbf{z}$ & $\mathbf{z}$ & $\mathbf{z}$ & & \\
\hline 298 & $11.4996(3)$ & $4.4454(3)$ & $4.1550(3)$ & $0.1195(1)$ & $0.101(1)$ & $0.8583(2)$ & $0.478(2)$ & $2.8(1)$ & 5.3 \\
\hline 348 & $11.5143(4)$ & $4.4409(3)$ & $4.1617(3)$ & $0.1197(1)$ & $0.100(1)$ & $0.8582(2)$ & $0.480(2)$ & $3.1(1)$ & 5.2 \\
\hline 398 & $11.5300(4)$ & $4.4368(3)$ & $4.1689(4)$ & $0.1199(1)$ & $0.097(1)$ & $0.8585(2)$ & $0.482(2)$ & $3.6(1)$ & 5.1 \\
\hline 448 & $11.5464(4)$ & $4.4294(4)$ & $4.1780(4)$ & $0.1201(2)$ & $0.094(1)$ & $0.8585(2)$ & $0.482(2)$ & $3.6(1)$ & 5.2 \\
\hline 498 & $11.5636(4)$ & $4.4216(4)$ & $4.1873(4)$ & $0.1203(2)$ & $0.091(2)$ & $0.8587(2)$ & $0.486(2)$ & $3.9(1)$ & 5.2 \\
\hline 548 & $11.5827(4)$ & $4.4127(4)$ & $4.1979(5)$ & $0.1205(2)$ & $0.091(2)$ & $0.8586(2)$ & $0.483(2)$ & $4.1(1)$ & 5.3 \\
\hline 598 & $11.6030(4)$ & $4.3996(4)$ & $4.2114(5)$ & $0.1210(2)$ & $0.086(1)$ & $0.8588(2)$ & $0.483(2)$ & $4.5(1)$ & 5.1 \\
\hline 648 & $11.6257(4)$ & $4.3854(4)$ & $4.2273(5)$ & $0.1216(2)$ & $0.079(1)$ & $0.8589(3)$ & $0.480(2)$ & $4.8(1)$ & 5.1 \\
\hline 698 & $11.6498(5)$ & $4.3664(4)$ & $4.2470(5)$ & $0.1222(2)$ & $0.069(2)$ & $0.8594(3)$ & $0.483(3)$ & $5.4(1)$ & 5.0 \\
\hline 748 & $11.6778(5)$ & $4.3400(4)$ & $4.2740(5)$ & $0.1231(2)$ & $0.055(2)$ & $0.8598(3)$ & $0.488(3)$ & $5.7(1)$ & 5.2 \\
\hline 798 & $11.7127(4)$ & $4.3010(4)$ & $4.3113(4)$ & $0.1241(2)$ & 0 & $0.8603(3)$ & $0.5(0)$ & $5.6(1)$ & 5.0 \\
\hline 848 & $11.7251(4)$ & $4.3034(3)$ & $4.3179(4)$ & $0.1242(2)$ & 0 & $0.8605(3)$ & $0.5(0)$ & $5.9(1)$ & 3.5 \\
\hline
\end{tabular}


Fig. S1. Close-up of X-ray powder diffraction patterns collected on bars cut from ingots 1 and 3.

Data are shown for faces parallel and perpendicular to the hot-press direction as illustrated in Figure 1 of the manuscript.

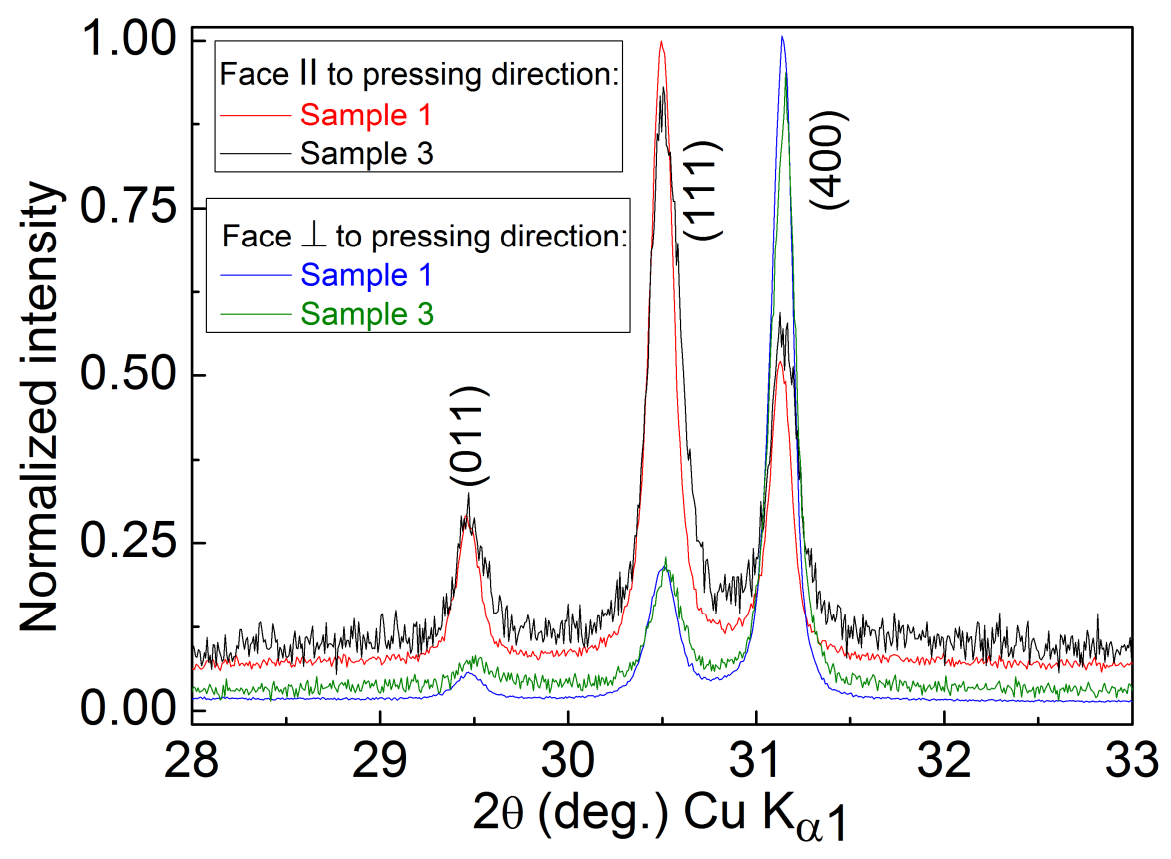


Fig. S2. SEM study of a bar cut parallel to the pressing direction from ingot 3 . This illustrates the presence of coherent domains of SnSe platelets oriented at $\sim 45$ degrees with respect to the hot-press direction. This coherent stacking of platelets is lost near the bottom surface of the pellet (image 4).
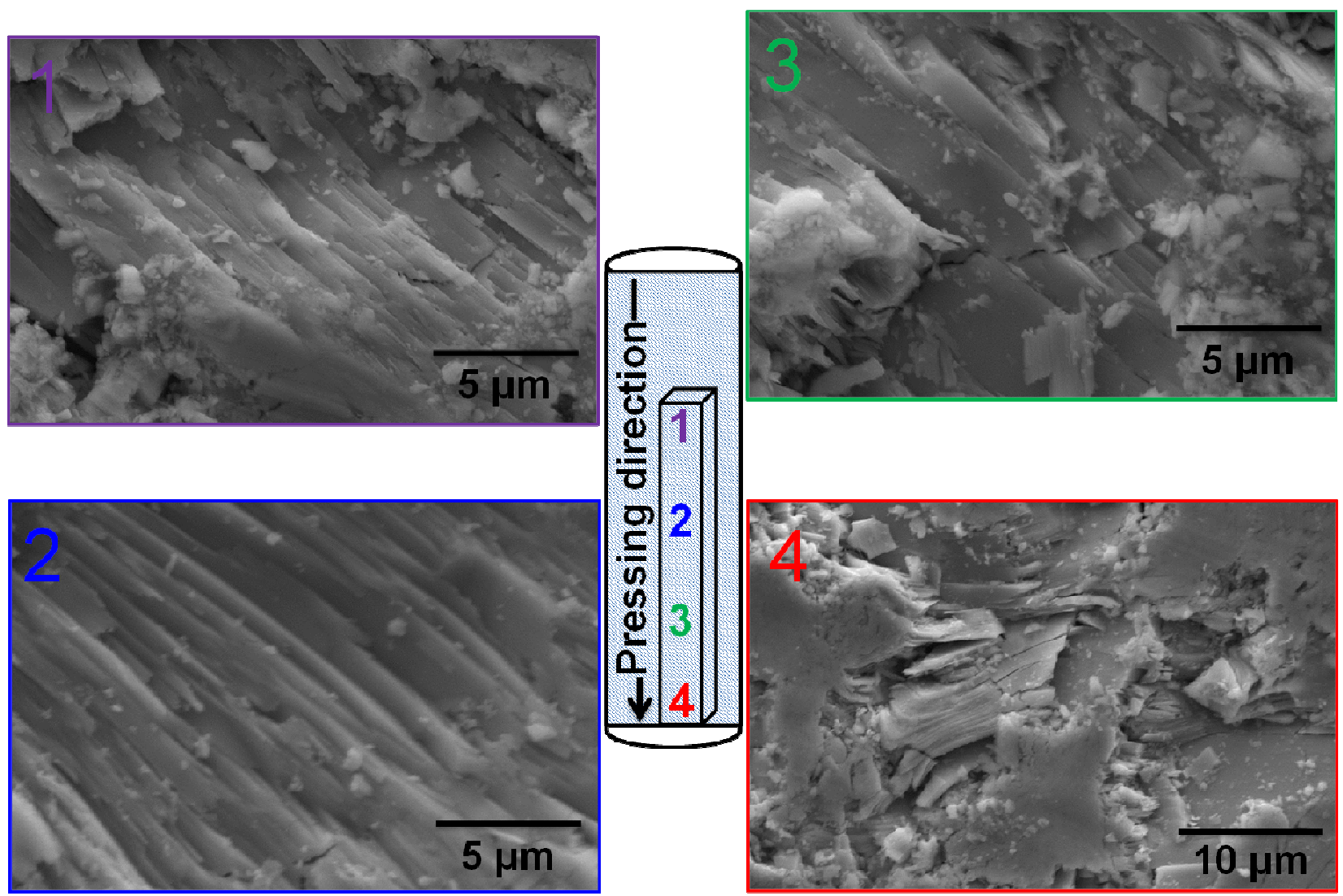
Fig. S3. Temperature dependence of the heat capacity of SnSe.

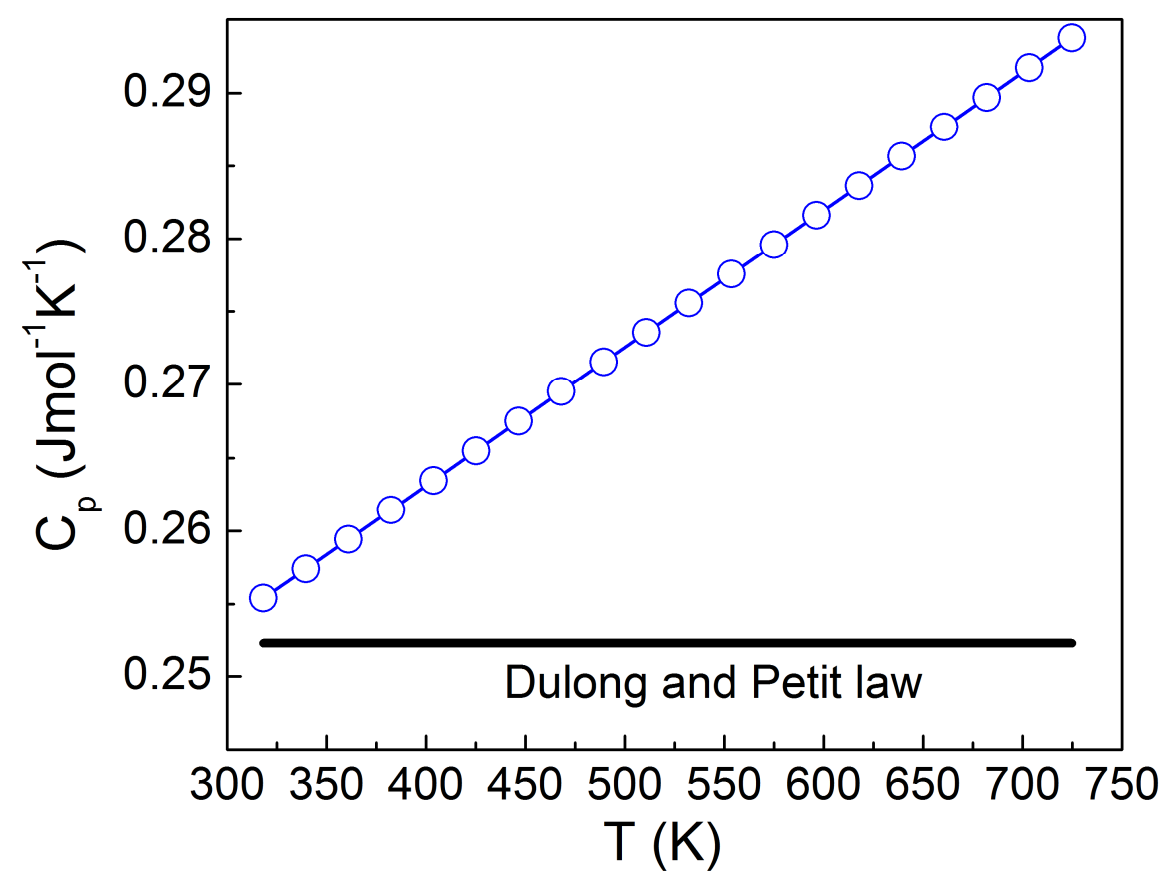


Fig. S4. Temperature dependence of the total $(\kappa)$, lattice $\left(\kappa_{\text {lat }}\right)$ and electronic $\left(\kappa_{\mathrm{el}}\right)$ thermal conductivity for SnSe. Data collected in the perpendicular to pressing direction, on a disc cut from ingot 3. $\kappa_{\text {lat }}$ was estimated using the Wiedemann-Franz law: $\kappa_{\text {lat }}=\kappa-L \sigma \mathrm{T}$, where $\mathrm{L}$ is the Lorenz number, $\sigma$ is the electrical conductivity, and $\mathrm{T}$ is the absolute temperature. $\left(\mathrm{L}=2.5 \times 10^{-8} \mathrm{~V}^{2} \mathrm{~K}^{-2}\right)$.

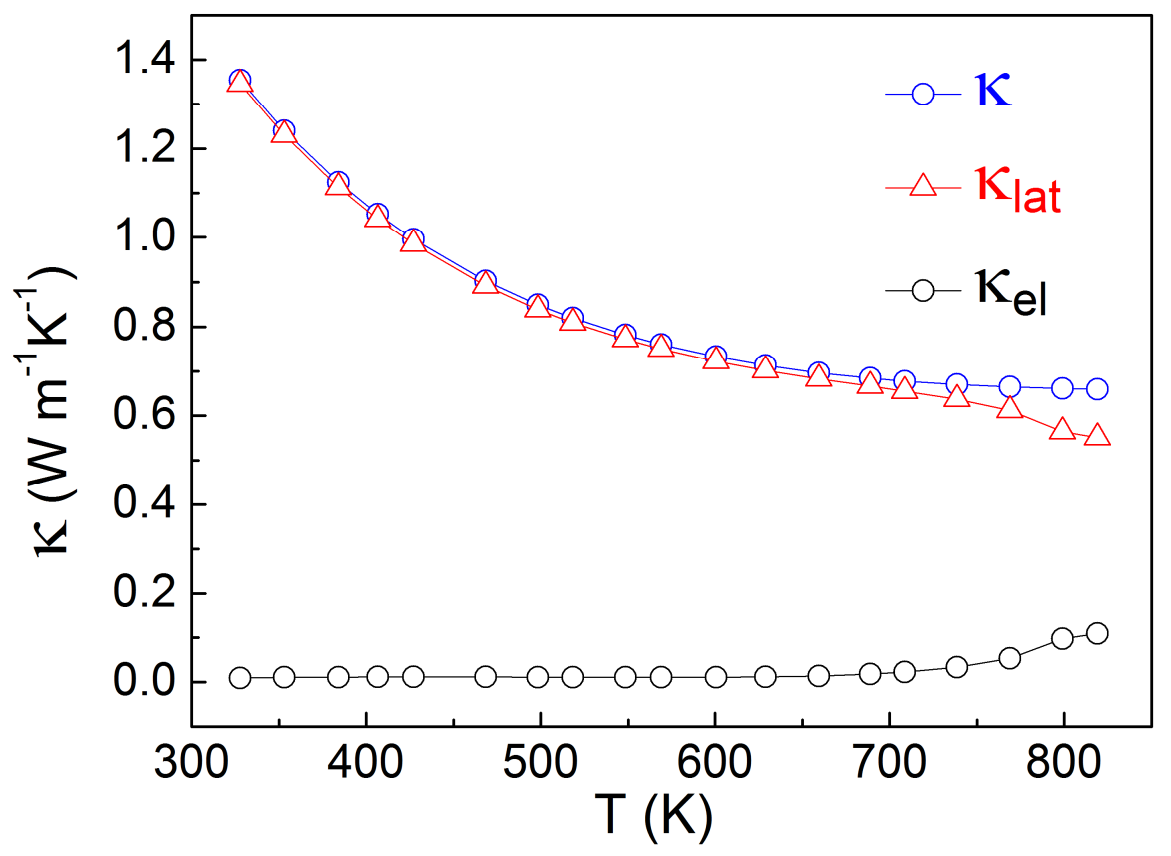


Fig. S6: Observed (open circles), calculated (red line) and difference (green line) Rietveld profiles for room temperature X-ray powder diffraction data collected on SnSe. Texturing was modelled using spherical harmonics preferential orientation coefficients. Data collected using a PANalytical Empyrean diffractometer (Mo $\left.\mathrm{K}_{\alpha 1, \alpha 2}\right)$.

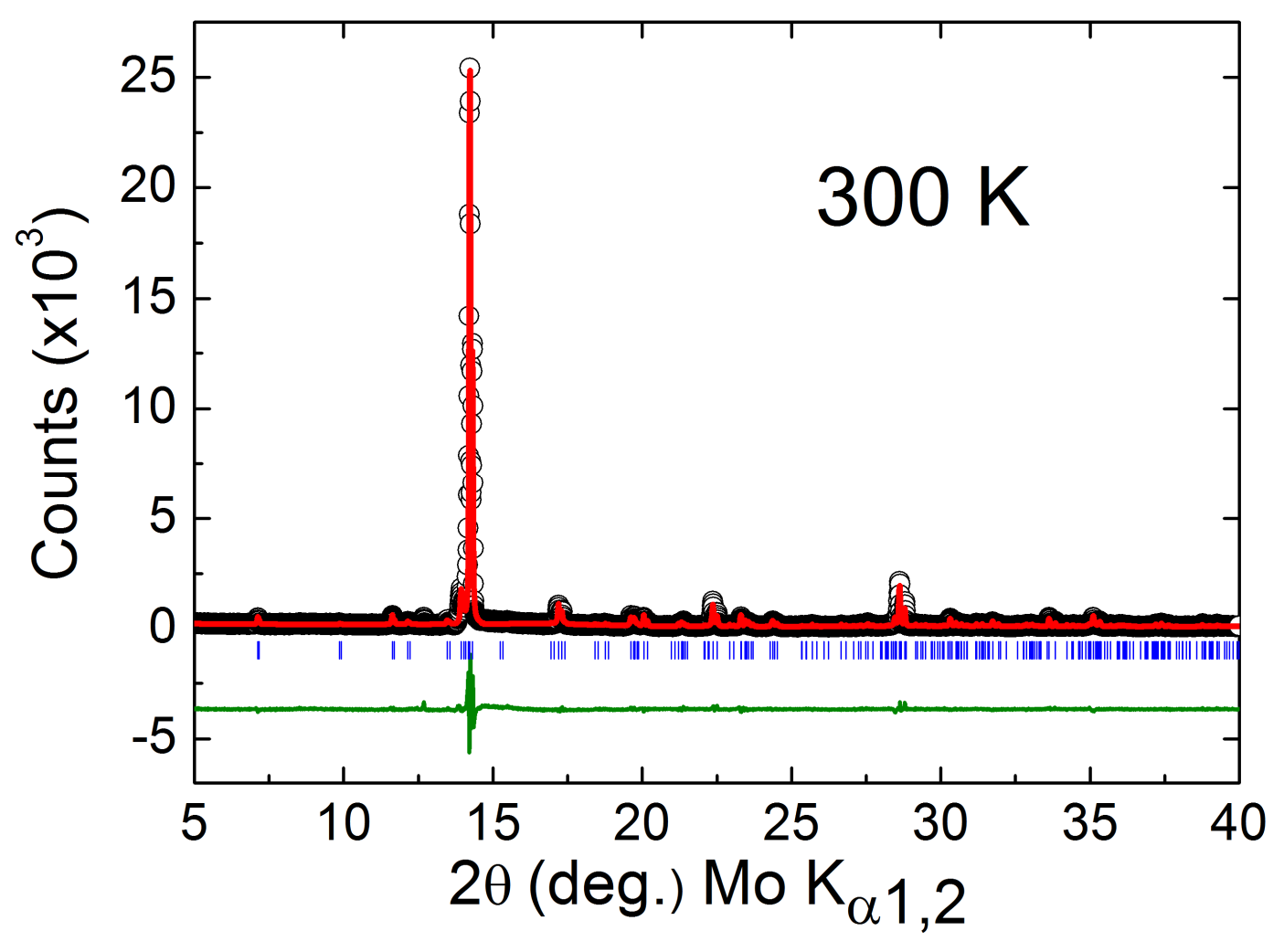

